Revista de Psicología de la PUCP. Vol. XIX, 2, 2001

\title{
Relaciones Objetales en niños maltratados y abusados a través del Rorschach y del MOA (Urist) ${ }^{1}$
}

\author{
Sylvia Rivera Carpio ${ }^{2}$ \\ Pontificia Universidad Católica del Perú
}

El objetivo fue conocer cómo es la calidad de las relaciones objetales en los niños y niñas institucionalizados, que han sido objeto de algún tipo de maltrato, a través del Psicodiagnóstico de Rorschach (Exner) y de la Escala de Mutualidad de Autonomía (MOA) de Urist. Los participantes fueron 23 niños de ambos sexos, entre 8 y 10 años que habían recibido maitrato en su entorno familiar. El grupo de estudio provino de los niños institucionalizados que se encuentran internados en un puericultorio, pertenecientes a un sector socio económico bajo. Los resultados mostraron que ambos instrumentos reflejan la calidad de las relaciones objetales de manera adecuada. Asimismo, existen diferencias significativas de acuerdo al sexo, momento de separación de la figura materna y tipo de maltrato recibido. Por otro lado, los menores de nuestro estudio logran representarse relaciones más adaptativas, convencionales y positivas que nos ligan con sus recursos.

Palabras claves: relaciones objetales, relaciones interpersonales, autopercepción, afectividad, maltrato, abuso, trauma, Rorschach, Exner, Escala de Mutualidad de Autonomía (MOA).

Object-relations in maltreated and abused children assessed with the Rorschach inkblots and the Mutuality of Autonomy Scale (Urist)

The objective of the study was to observe the quality of the object-relations in maltreated and institutionalized boys and girls, assessed with the Rorschach inkblots and the Mutuality of Autonomy Scale (MOA) of Urist. The participants were 23 children of both sexes, aged 8 to 10 , who had been maltreated in their household and were institutionalized in an orphanage. The results showed that both tools reflect object-relations well. There are significant differences, depending on the sex, time of Separation from their maternal figure and the type of maltreatment they received. Furthermore, the children represent more adaptative, conventional and positive object relations what is related with their resources.

Keywords: object-relations, interpersonal relations, self-perception, affectivity, maltreatment, abuse, trauma, Rorschach, Exner, Mutuality of Autonomy Scale (MOA).

1 Una versión de este trabajo fue presentado en el XI Congreso Latinoamericano de Rorschach y otras técnicas proyectivas celebrado en Rosario en mayo de 2001.

2 Licenciada de Psicología de la Pontificia Universidad Católica del Perú y Miembro de la Sociedad Peruana de Rorschach. Actualmente se encuentra trabajando en el Hogar de niños abandonados "San Luis" de Chorrillos. Correo electrónico: riveval @terra.com.pe. 

Aún en el siglo XXI, el maltrato y abuso por parte de las figuras más cercanas y significativas del niño se erigen como unas de las vivencias más traumáticas a las que este se ve expuesto. Por este motivo, la psicología ha estudiado a lo largo de muchos años, este tema centrándose en las características de personalidad tanto de la víctima y del agresor, como en las consecuencias del mismo. Lo que motivó e impulsó este estudio es, por un lado, la carencia de investigaciones respecto al maltrato y abuso que aborden el impacto de estos en los sistemas y estructuras psicológicas tales como funciones yoicas y relaciones objetales. La segunda motivación está dada por el empleo de ambos instrumentos: el Psicodiagnóstico de Rorschach, según el S. C. de Exner (1994) junto al empleo de la Escala de Mutualidad de Autonomía (MOA) de Urist, ya que creemos que ambos pueden brindarnos un aporte en la descripción de las relaciones objetales.

Las relaciones objetales son las imágenes internalizadas que los seres humanos tenemos de aquellas personas que resultan significativas para nosotros. De acuerdo con estas imágenes y representaciones internas es que nosotros nos desenvolvemos e interactuamos con el entorno. El maltrato es toda aquella agresión o daño producida al niño por sus padres, hermanos, familiares, maestros y otras personas a pesar de haber sido hecho con la intención de disciplinarlo, produciéndose también maltrato cuando no se atienden las necesidades de alimentación, protección, afecto y cuidado del niño.

Todo maltrato tiende a producir un trauma que debe ser elaborado, ya que de no ser así, queda como quiste residual, dinámico y vivo, que trata de incorporarse al sistema; y que al no poder disolverse, regresa interactivamente en forma de idea o de acción. De idea, como en los sueños y de acción, en la repetición de lo vivido por persona interpuesta: se escenifica con otro para tratar de procesar el recuerdo; lo cual lleva a que el maltratado repita su experiencia con 
otro, dando lugar a un proceso inacabable de víctima-víctimario. Así, siguiendo a Pine (1990), los intentos por dominar y elaborar las experiencias traumáticas que se han ido acumulando, generan la tendencia a repetir las experiencias abusivas internalizadas.

El maltrato, así como el abuso, genera una rabia muy intensa que necesita ser expresada. Además, podemos notar que la capacidad de confianza en el otro y sobre todo en el adulto se ve fuertemente limitada a partir del maltrato; los menores abusados se muestran distantes, herméticos y poco comunicativos, por lo que resulta muy difícil poder ganar su confianza en la relación interpersonal.

La Teoría de las Relaciones Objetales sostiene que las relaciones más tempranas establecen los cimientos para la creación gradual de los modelos representacionales que guían las relaciones subsiguientes y, en última instancia, las que se establecen con los propios hijos. Lo que interesa a esta teoría (Greenberg y Mitchell, 1983) es que la cantidad de experiencias de una persona con otras va tomando forma de acuerdo con un patrón de relaciones que incluye un modelo del otro y que —en lenguaje cotidiano - es llevado en la cabeza para siempre. Estas imágenes internas constituyen un residuo interno de aquellas relaciones con las personas importantes en la vida de individuo, que dan forma a las subsecuentes actitudes, reacciones, percepciones, etc.

Consideramos que el estudio de las relaciones objetales nos abre un amplio espectro de posibilidades para observar las relaciones que el niño establece y así plantear alternativas y acciones que contribuyan a disminuir la frecuencia del maltrato o a mediar sus efectos. Por este motivo, la Teoría de las Relaciones Objetales resulta un paradigma muy apropiado para ilustrar acerca de cómo y por qué diferentes tipos de abuso influyen de manera diferencial en una dimensión del funcionamiento de la personalidad.

El Psicodiagnóstico de Rorschach es un método proyectivo que le posibilita al evaluado externalizar sus propios contenidos y su 
mundo privado a través de una amplia gama de respuestas posibles. Por esto, O. Kernberg (1976) propugnó para que las nacientes teorías de la relación con el objeto generen nuevos enfoques en el uso del Rorschach, al vincular de manera bastante estrecha las funciones del yo y las relaciones objetales. Es en este sentido, que Smith (1994) sostiene que la Teoría de las Relaciones Objetales resulta un marco teórico muy adecuado para la interpretación de los datos del Rorschach, ya que la personalidad y la psicopatología son concebidas en términos de relaciones con objetos, tanto externos como internos.

Ha sido sobre la base de estas consideraciones que nos hemos propuesto realizar un estudio cuyo objetivo general es conocer cómo es la calidad de las relaciones objetales en los niños y niñas institucionalizados entre 8 y 10 años, que han sido objeto de algún tipo de maltrato, a través del Psicodiagnóstico de Rorschach, según el Sistema Comprehensivo de Exner y de la Escala de Mutualidad de Autonomía (MOA) de Urist.

Existen antecedentes de investigaciones realizadas con relación al tema, como es el caso de Kelly (1999) que ha realizado un estudio con niños abusados y traumatizados que emplea tanto el Rorschach infantil como la Escala de Mutualidad de Autonomía (MOA) de Urist. Adicionalmente, en el Perú contamos con el estudio de Garrido-Lecca (1999) que examina las relaciones objetales en menores desplazados, empleando el Psicodiagnóstico de Rorschach.

Consideramos relevante la ejecución del presente estudio por ser uno de los pocos que se realizan en el área de la psicología clínica y del desarrollo en nuestro medio, y por permitir evaluar el efecto del maltrato y abuso en los sistemas intrapsíquicos tales como el funcionamiento yoico y las relaciones objetales. 


\section{Metodología}

El estudio pertenece a un nivel exploratorio, ya que se trata de estudiar la calidad de las relaciones objetales que presentan los niños de 8 a 10 años de edad de ambos sexos, que han sufrido maltrato físico o abuso sexual y que se encuentran institucionalizados. Adicionalmente, las investigaciones acerca del tema del maltrato con respecto a las relaciones objetales aún resultan escasas en nuestro medio.

\section{Participantes}

Los participantes fueron 23 niños de ambos sexos, entre 8 y 10 años que habían recibido maltrato en su entorno familiar. El grupo de estudio provino de los niños institucionalizados que se encuentran internados en un puericultorio, pertenecientes a un sector socio-económico bajo. No se empleó un segundo grupo equivalente, debido principalmente a la dificultad para definir al grupo de comparación, ya que los niños de la muestra presentan dos características que los distinguen y particularizan: el maltrato y la institucionalización. Este grupo fue obtenido a través de un procedimiento de muestreo no probabilístico de tipo intencional (Rivera Carpio, 2000).

\section{Instrumentos}

El Psicodiagnóstico de Rorschach. Esta técnica proyectiva le permite al sujeto externalizar sus propios contenidos y su mundo privado, a través de una amplia gama de respuestas posibles. Tiene como propósito principal, proporcionar una descripción clínicamente significativa de la personalidad de un individuo y brinda información sobre aspectos o áreas fundamentales de su organización, como los cognitivos o intelectuales, los afectivos o emocionales, y los referentes al funcionamiento del ego, dentro de los cuales nos centraremos en la calidad de las relaciones objetales. 
Como ya señalamos, el Rorschach nos brinda información muy significativa acerca del individuo en diferentes aspectos, entre los cuales están las relaciones objetales, estrechamente vinculadas con las relaciones interpersonales, así como con otros signos o indicadores propios del Psicodiagnóstico de Rorschach según el Sistema Comprehensivo de Exner. Adicionalmente, existen algunos signos que resultan esenciales para la interpretación desde la Teoría de las Relaciones Objetales como es el estudio del movimiento, tanto humano como animal, acompañado por pares, y que constituyen los signos más relevantes del Psicodiagnóstico de Rorschach para evaluar tales relaciones, ya que las expresan de manera particularmente vívida y personal (Rosegrant, 1984).

La Escala de Mutualidad de Autonomía (MOA) de Urist. El MOA evalúa el contenido temático de las respuestas de movimiento del Rorschach, puntuando todas las respuestas de movimiento, ya sea humano, animal o inanimado a lo largo de una escala continua ordinal de 7 puntos. Este continuo se inicia en el nivel 1 en el que las respuestas connotan relaciones positivas, empáticas, separadas y autónomas, y finaliza en las respuestas de grado 7 , que indican la representación de relaciones caracterizadas por ser malevolentes, con miedo de incorporación, envolventes, etcétera. Los resultados generales pueden proveer de importante información acerca de las representaciones objetales del individuo. Los estudios reportan coeficientes de validez y confiabilidad razonables tanto en grupos normales como clínicos (Urist, 1977), oscilando la confiabilidad entre 0.73 y 0.90 (Meyer y Tuber, 1989; Ryan, Avery y Grolnick, 1985). En nuestro estudio, se estableció la validez de contenido del MOA, para cada nivel de la escala, observándose que estos niveles oscilaban entre 0.71 y 1.00, resultando aceptables para la validación de la escala (Spector, 1992). 


\section{Procedimiento}

Una vez obtenido el consentimiento de la directora del puericutorio, se solicitaron entrevistas con las psicólogas encargadas de la sección, planteándoseles los criterios de inclusión y revisándose las fichas de ingreso de los menores, con lo que estableció contacto con cada uno de los participantes en la investigación. Se procedió a la aplicación individual del Psicodiagnóstico de Rorschach y de la ficha socio demográfica a cada miembro de la muestra. Luego se codificó cada protocolo según los criterios de Exner, realizándose el vaciado de datos en el Sumario Estructural de cada caso a través del programa computarizado (RSP3), y posteriormente se realizaron los análisis estadísticos mediante el programa SPSS.

\section{Resultados}

Presentaremos los resultados organizándolos en tres ejes fundamentales de análisis: el primero se refiere a la descripción de las características propias del grupo a través del Psicodiagnóstico de Rorschach; el segundo, describe la calidad de las relaciones objetales a través del MOA; y el tercero hace alusión a la interrelación de los diversos aspectos de estudio.

Para el análisis estadístico, se realiza la comparación de medias con los datos normativos mediante la prueba $t$ de Student. Dado que la muestra es pequeña y las variables indican distribuciones no normales, se utiliza el análisis de varianza no paramétrico de Kruskall Wallis y la prueba de comparación de medianas U de Mann Whitney. Se trabaja con niveles de significación de 0.05 y 0.10 para ambos casos.

Con respecto a lo hallado en las Relaciones Interpersonales (Cuadro 1), encontramos que la mayoría de niños maltratados (73.9\%) presenta un interés por establecer vínculos (revelado a partir de las respuestas M y (2)). Sin embargo, estas relaciones se caracterizan por 
ser algo distantes y superficiales (revelado por los tipos de respuestas de Contenidos Humanos, Interés interpersonal 34.8\%). Esta distancia se encuentra ligada con la dificultad para relacionarse de manera cálida y cercana (ausencia de respuestas $\mathrm{T} 52 \%$ ) al resultar los acercamientos del otro amenazantes.

\section{Cuadro 1}

Diferencias al interior del grupo de niños maltratados en el área interpersonal

\begin{tabular}{|lllccc|}
\hline $\begin{array}{l}\text { Variables de R. } \\
\text { Interpersonales }\end{array}$ & Sexo & Edad & N. Intelectual & $\begin{array}{c}\text { Momento de } \\
\text { Separación }\end{array}$ & $\begin{array}{c}\text { Tipo de } \\
\text { Maltrato }\end{array}$ \\
\hline COP & $.047^{*}$ & - & - & - & - \\
AG & - & - & - & - & - \\
Fd & - & - & - & $.015^{*}$ & - \\
SumH & - & - & - & - & - \\
M y (2) & - & - & - & $-025^{*}$ & - \\
FM y (2) & - & - & - & - & $.0743^{* *}$ \\
T & - & - & - & & \\
\hline
\end{tabular}

$* p<.05 \quad * * p<.10$

La fantasía estaría sirviendo como una defensa en nuestros menores para no sentir esta amenaza frente al otro, quedándose generalmente con los aspectos más idealizados y positivos del otro para así mantener cierto equilibrio interno, percibiéndose a sí mismos y a los otros de manera imaginaria, parcial y escindida ( $\mathrm{Hd}+\mathrm{Ad}, 52.2 \%)$.

Al interior del grupo, observamos que la mayoría de varones $(66.7 \%)$ presenta una tendencia a establecer vínculos positivos y cooperativos (COP). Las niñas, en cambio, estarían invirtiendo una mayor cantidad de energías y tiempo en interpretar las intenciones del otro, en el acercamiento, mientras que los varones responden de manera más inmediata (FM) a las situaciones sociales.

La separación temprana de la figura materna establece diferencias y tendencias significativas, observándose que la mayoría de niños $(50 \%)$ que fue separado antes de los 3 años, presenta una mayor necesidad de 
recibir atención y cuidado (Fd) al no haberse visto protegidos y atendidos tempranamente.

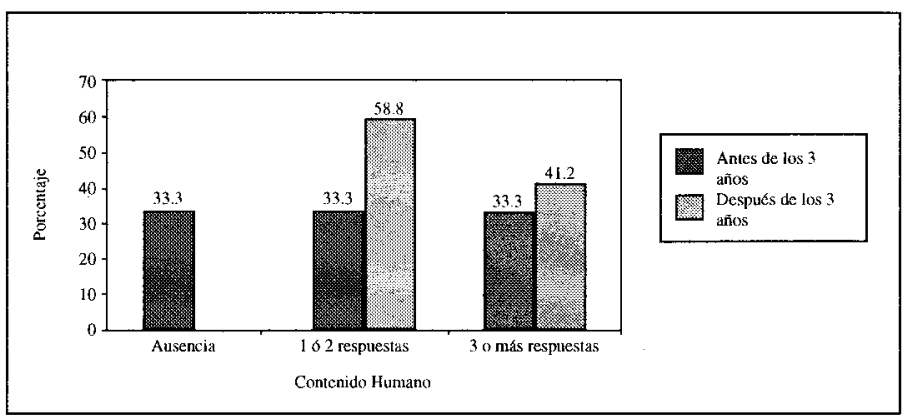

Figura 1. Contenido humano por momento de separación.

En la Figura 1, se puede observar que la ausencia de respuestas de contenido humano en un $33.3 \%$, corresponde al grupo que fue separado de la figura materna antes de los 3 años, que muestra una escasa motivación para establecer relaciones con los demás, así como desapego y desinterés.

En cuanto a lo observado en las relaciones interpersonales de los niños que han sido separados tempranamente, observamos que a pesar de esta marcada necesidad afectiva, nuestros menores estarían poco interesados en el contacto y relación con el otro (ausencia de H) y se rigidizarían, mostrándose poco espontáneos (FM y (2)) en el contacto interpersonal, generando un aumento de la conducta de aislamiento y una falta de empatía que surge a partir de la destrucción temprana de vínculos humanos que no han posibilitado que estas cualidades se hayan desarrollado. La capacidad de confianza en el otro se ha visto limitada en la mayoría de menores separados tempranamente $(50 \%)$ ( $\mathrm{Hd}+\mathrm{Ad}$, Respuestas para-humanas para animales), remitiéndonos a una percepción parcial y escindida de los objetos, limitando esto una relación más real con el otro y una mayor integración de sí mismo. La ausencia de la figura materna expondría al niño 
Relaciones objetables en niños maltratados y abusados a través del Rorschach y del MOA

ante un mayor riesgo traumático ya que esta cumple la función de desempeñarse como una barrera temporal, posibilitándose el desarrollo de los recursos yoicos del niño.

El tipo de maltrato establece diferencias al interior del grupo, observándose que las niñas abusadas prefieren evitar el contacto y cercanía con el otro (Ausencia de Respuestas $\mathrm{T}$ ) a partir de la pérdida de confianza en el adulto al haberse visto traicionadas, alertándose ante el acercamiento del otro. Por otro lado, los niños maltratados estarían idealizando al maltratador $((\mathrm{Hd})+(\mathrm{Ad}) 60 \%)$ para preservarlo y así mantener cierto equilibrio.

Con respecto a la Autopercepción (Cuadro 2), observamos una marcada devaluación en la gran mayoría del grupo (índice de Egocentrismo $82.6 \%$ ) al no poder responder a las exigencias del entorno y que lleva a una parte de este grupo al retraimiento ( $\mathrm{H}+\mathrm{A}<\mathrm{Hd}+\mathrm{Ad}$ $17.4 \%$ ), sintiéndose rechazados por el otro. La percepción de sí mismos se encuentra dañada y desvalorizada (MOR 30.4\%) generando depresión e interfiriendo con el interés en relaciones interpersonales más profundas. El maltrato y abuso han generado una sensación de indefensión y un temor al daño corporal que se traducen en mayor preocupación por el cuerpo (An+ Xy 43.5\%).

\section{Cuadro 2}

Diferencias al interior del grupo de niños maltratados en Autopercepción

\begin{tabular}{|lccccc|}
\hline $\begin{array}{l}\text { Variables de } \\
\text { Autopercepción }\end{array}$ & Sexo & Edad & N. Intelectual & $\begin{array}{c}\text { Momento de } \\
\text { Separación }\end{array}$ & $\begin{array}{c}\text { Tipo de } \\
\text { Maltrato }\end{array}$ \\
\hline $3 \mathrm{r}+(2) / \mathrm{R}$ & - & - & - & - & - \\
FD & - & - & - & - & - \\
Vista & - & - & - & - & $.09^{*}$ \\
An+Xy & $.076^{* *}$ & - & - & - & - \\
MOR & $.095^{* *}$ & - & - & - & .049 \\
\hline
\end{tabular}

${ }^{*} p<.05{ }^{* *} p<.10$ 
Al interior del grupo, se puede observar que las niñas presentan una mayor amenaza de abuso sexual, expresándose esto en un aumento de la preocupación corporal (An+Xy 57.1\%) El maltrato ha generado una percepción un tanto más dañada y desvalorizada en este grupo (MOR 42.9\%) que se estaría relacionando con tres aspectos en los cuales nos detendremos posteriormente.

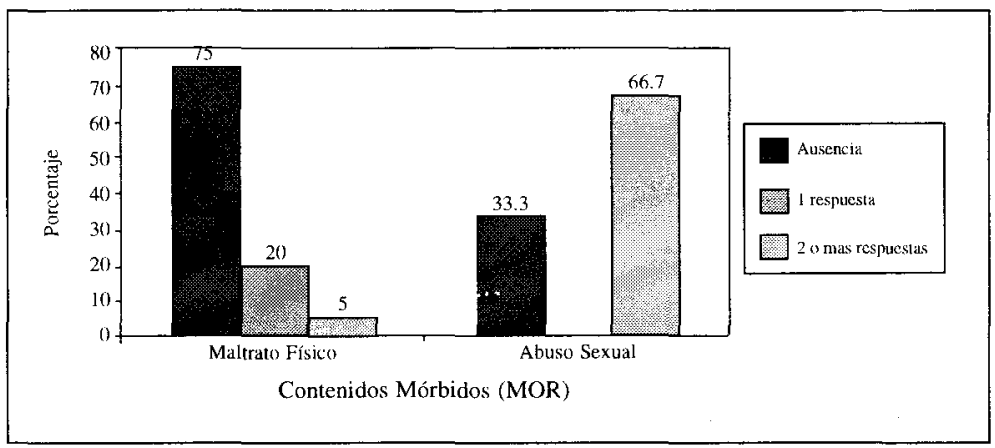

Figura 2. Contenidos mórbidos por tipo de maltrato.

En la Figura 2, podemos observar que el tipo de maltrato establece diferencias al interior del grupo, ya que las niñas abusadas presentan una sensación más intensa de daño y una percepción más pesimista de la realidad (MOR 66.7\%). Asimismo, este grupo presenta una fuerte autocrítica negativa (V), que surgiría a partir de la estigmatización, generando culpa y vergüenza.

Con relación al Afecto, se pudo observar que el temor y desconfianza ante la expresión afectiva lleva a nuestros menores a controlarlo de manera rígida (Proporción afectiva $<78.3 \%$ ), generándose un malestar (lado derecho de eb $>52.2 \%$ ) que no es superado en la medida en que no se viabilice la descarga.

En la Figura 3, podemos observar que el tipo de maltrato establece diferencias al interior del grupo, ya que las niñas abusadas reprimen y controlan su posibilidad de mostrar afectos (FC) para así no 
verse amenazadas. Sin embargo, se les dificulta la experiencia y expresión afectiva ya que en este intento de control, aislamiento y contención, estas vivencias se expresan de manera un tanto brusca e inmodulada (C), en especial ante aquellas situaciones cargadas afectivamente.

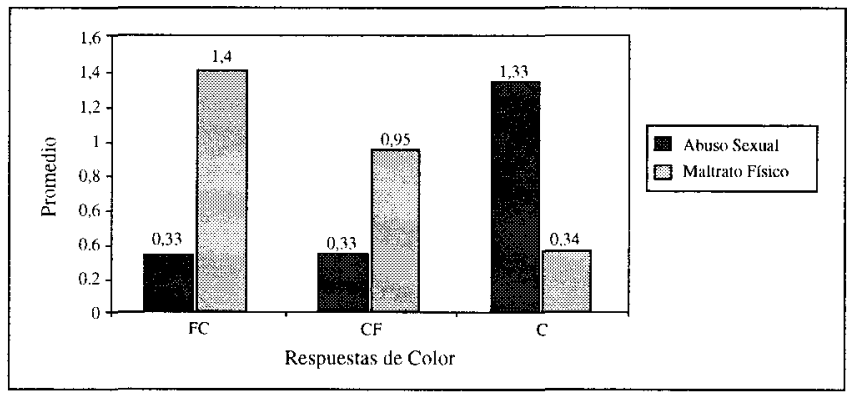

Figura 3. Respuestas de color por tipo de maltrato.

Con respecto a nuestro segundo eje que examina las diferencias y tendencias halladas a través de la escala de Mutualidad de Autonomía (MOA), observamos de modo general, que la mayoría de nuestros casos se representa relaciones marcadas por un componente de control, daño y destrucción (LOR 5, 6 ó 7, 58\%) que se relaciona con cierto temor al ataque. Por otro lado, la gran mayoría de nuestro grupo (HOR 1 ó 2,89\%) tienen la posibilidad de representarse relaciones adaptativas, convencionales, diferenciadas y positivas, balanceando así las representaciones desorganizadas con aquellas más bien adaptativas.

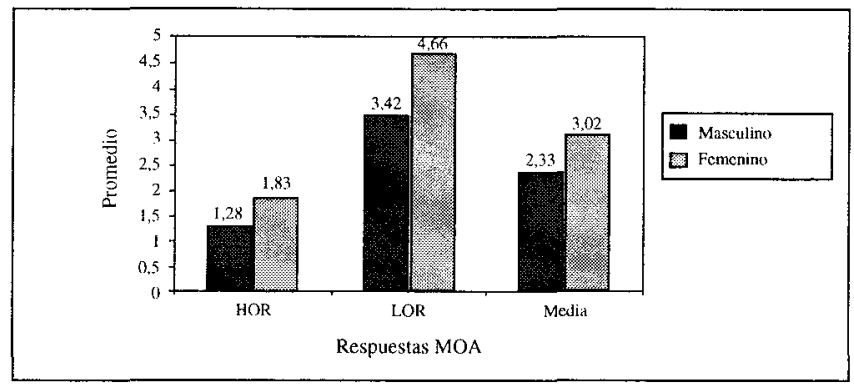

Figura 4. Respuestas MOA por sexo. 
$\mathrm{Al}$ interior del grupo, observamos (Figura 4) que los varones se representan las relaciones de manera más benigna, integrada y adaptativa (HOR) en la que cada uno de los miembros se muestra más autónomo. Por su lado, las niñas presentan representaciones de relaciones más malevolentes (LOR) y en general se representan las relaciones de manera más distorsionada (MediaMOA), ubicándose en una posición más desvalida e indefensa que los varones, dentro de relaciones coercitivas de víctima y victimario.

La separación temprana ha influido notablemente en todo el grupo de niñas, pues - como ya señalamos anteriormente - la gran mayoría del grupo $(83.3 \%)$, que fue separado prematuramente de la madre fueron mujeres. Para Lynch y Cicchetti (1991), los problemas de apego primario generan distorsiones en las relaciones que se acompañan de representaciones mentales negativas, originando patrones confusos en la relación de las niñas con sus madres, que pueden persistir a lo largo de la latencia, en la cual se vuelven más vulnerables (Monahon, 1993). Si la separación de la madre, tiene consecuencias graves tanto para los niños como para las niñas, esta situación se ve agravada al ser separadas estas últimas de su figura materna, ya que se ve afectada la capacidad empática, así como la sensibilidad y la conexión con el otro que definen a la niña en su proceso identificatorio.

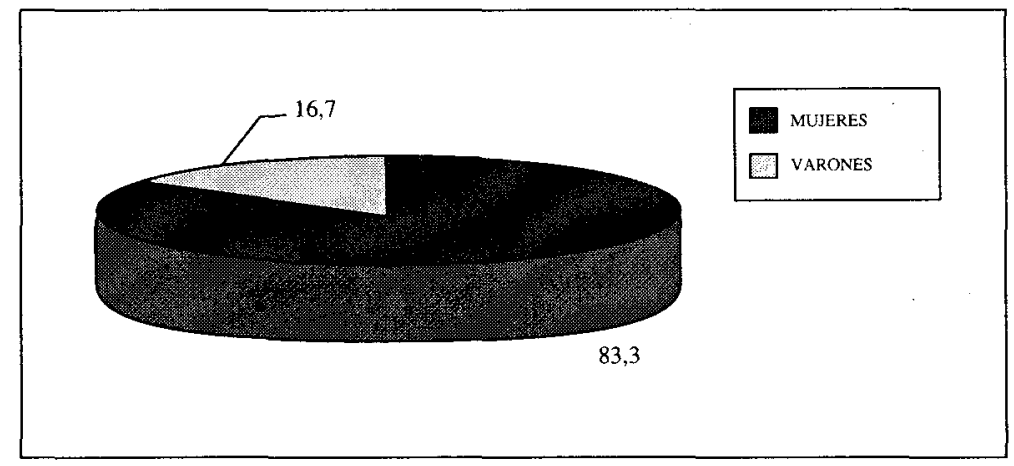

Figura 5. Proporción de niños que fueron separados tempranamente de la madre. 
El momento de separación de la figura materna no establece diferencias significativas en función al MOA, principalmente debido a que el SC tiene la capacidad de discriminar tanto aspectos estructurales como dinámicos de la personalidad, mientras que el MOA enfatiza principalmente en aspectos cualitativos y de funcionamiento; esto refleja que a pesar de la separación temprana, este grupo de niños cuenta con la posibilidad de establecer relaciones diferenciadas, autónomas y convencionales aunque algo superficiales y no muy estrechas.

El tipo de maltrato marca diferencias al interior del grupo ya que el abuso genera un efecto muy intenso que en ocasiones resulta devastador, presentando las niñas abusadas una tendencia a representarse relaciones marcadas por un mayor componente de daño y destrucción (Nivel 7 MOA).

Con relación a nuestro tercer eje que se refiere a la interrelación de ambos instrumentos, observamos que el poder definirse a sí mismo como alguien diferenciado, separado y autónomo (N2) va a permitir que el menor pueda establecer relaciones positivas (COP). Al percibir el niño que puede ser dañado o controlado por el otro en la relación (N5), tenderá a mantener una actitud más defensiva y agresiva con el otro (AG).

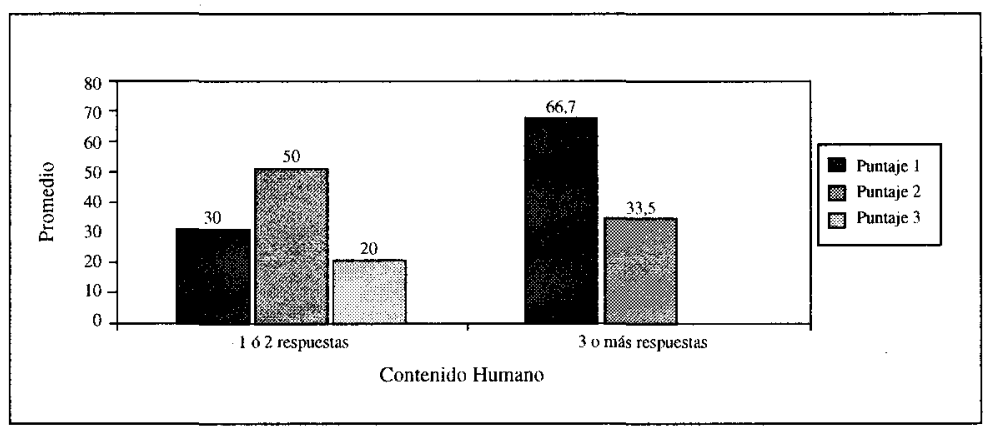

Figura 6. Contenido humano por puntaje más benevolente (HOR). 
El percibirse y valorarse positivamente (índice de Egocentrismo) le permitirá al niño relacionarse de manera adecuada, benevolente y con seguridad (HOR). Además, si el niño logra percibirse a sí mismo y a los otros a partir de las características que lo diferencian y definen $(\mathrm{H})$, podrá relacionarse de manera recíproca y diferenciada. (HOR) (Figura 6).

En la Figura 7, podemos observar que al percibir al otro y a sí mismo de manera idealizada o devaluada $((\mathrm{H})+(\mathrm{A}))$, el niño se representará relaciones en las que uno de los miembros aparece como muy poderoso y controlador (LOR).

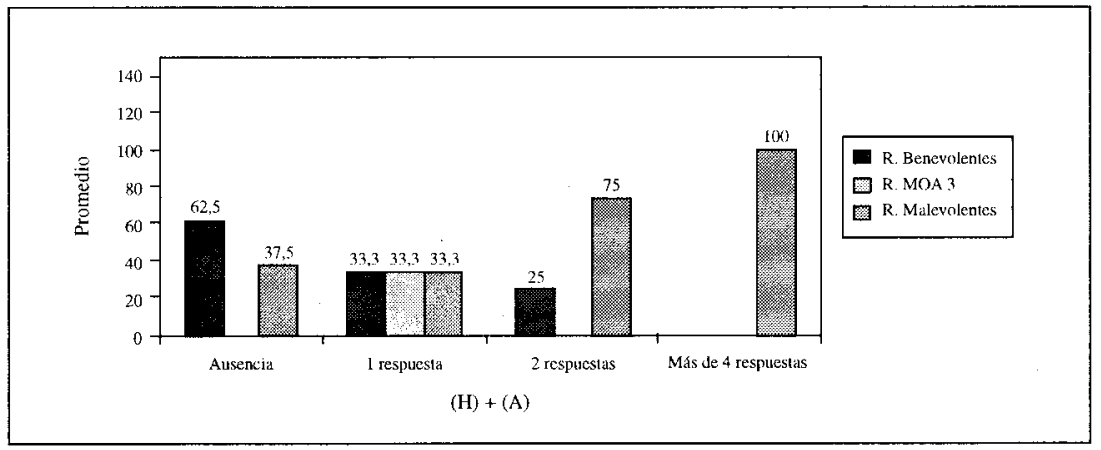

Figura 7. Percepciones parcializadas por respuesta más malevolente.

Por último, podemos señalar que al percibir que la relación con el otro resulta amenazante (LOR), el niño puede tender a defenderse conscientemente, intentando no exteriorizar el afecto (SumC').

\section{Discusión}

Conociendo que los primeros años de vida son esenciales para el desarrollo posterior, ya que sientan las bases para los logros de las etapas subsiguientes; nos preguntamos cuán fuertes pueden ser las huellas dejadas por el maltrato y el abandono en las relaciones objetales 
que estos niños han ido construyendo, sobre la base de los diversos modos de vinculación con sus seres significativos, en donde ha primado la indiferencia, violencia y/o abuso.

Consideramos que esta es una línea que no ha sido lo suficientemente explorada, a pesar de su importancia, porque nos puede abrir muchas posibilidades en el campo clínico y sobre todo en la prevención, tanto de la población infantil como de adultos en riesgo. Creemos además, que es parte de nuestro compromiso comprender a estos niños, explorar sus vivencias y sostenerlos, con el objetivo de ayudarlos a construir un espacio interno de ilusión. Este logrará constituirse únicamente si se les provee de objetos maternantes, que les posibiliten una experiencia exitosa, lo cual devendrá, con el tiempo, en un sentimiento básico de confianza y en la obtención de una mayor autonomía (Valeros, 1997).

Las relaciones objetales son aquellas imágenes internas que constituyen un residuo de la interacción con personas importantes en la vida del individuo. Estas relaciones tempranas proveen de una base para la creación gradual de modelos representacionales que guíen las subsiguientes (Levine y Tuber, 1993).

La experiencia del maltrato replantea los patrones de relación en el niño, viéndose que la posibilidad de confianza en el otro estaría claramente afectada, así como la creencia que el niño tiene acerca de su propia valía (FM y 2, y ausencia de H). La Teoría de las Relaciones Objetales provee una formulación de manera explicativa de la perpetuación del círculo vicioso del maltrato, ya que plantea que los patrones repetitivos en la vida tienden a persistir, debido a la clara tendencia a preservar la continuidad, familiaridad y conexión del mundo interpersonal, a pesar de lo patológico que esto puede resultar, pues el abuso y el trauma generan una secuela que envuelve la tendencia universal a repetir las viejas relaciones objetales (Pine, 1990). 
Tomando en cuenta entonces que la relación vincular con la madre sirve como un patrón que guía y dirige las relaciones subsiguientes del niño (Pine, 1990), no es de extrañar que la experiencia de discontinuidad e interrupción de la relación con esta, provoque en el menor, no solo la pérdida de interés por ella, sino también por los otros; así se debilita consecuentemente su proceso identificatorio, al no lograr establecer vínculos significativos con los demás.

La prevalencia de Detalles Humanos y Animales (Hd+Ad) en los menores que han sido separados tempranamente de su figura materna, nos remite a una tendencia a percibir a los objetos de manera parcial y escindida. En este sentido, prima un funcionamiento prioritariamente esquizo-paranoide, sin poder acceder a una vivencia más integrada del objeto (posición depresiva). La imposibilidad de integrar la percepción del otro como objeto total, dificulta la elaboración y reparación de las pérdidas internas y adicionalmente restringe las posibilidades de construir una imagen integrada de sí mismo.

La variable Momento de separación de la figura materna marca, entonces, diferencias importantes entre el grupo que fue separado de esta antes de los tres años y aquel cuya separación fue posterior, donde el primero es intensamente más afectado. Observamos hasta ahora, que a pesar de su gran demanda, los niños separados antes de los 3 años, buscan aislarse y no presentan interés por relacionarse con el otro, surgiendo en ellos una sensación de soledad, desesperanza y aislamiento, ya que las relaciones no pueden contribuir a enriquecerlos. Adicionalmente, en este grupo, la fuerte suspicacia y desconfianza en las relaciones sociales, al no haber podido desarrollar capacidad de confianza en el otro, unida a las características ya mencionadas, evidencian la importancia central del primer vínculo, que brinda un patrón que afecta y guía las relaciones subsecuentes.

En este sentido, debemos tener en cuenta que las relaciones tempranas cercanas sirven como un factor protector para la adversidad o estrés posterior. La madre cumpliría la función de desempeñarse 
como una barrera temporal ante los estímulos, mientras que se van desarrollando los recursos yoicos del niño, para así poder prevenir y evitar el trauma. Así, si el niño se ve expuesto a situaciones particularmente estresantes y traumáticas tempranamente y ya ha sido separado de su figura materna, se encontrará en mayor riesgo que si hubiera permanecido durante un período más prolongado cerca de la misma.

Como un factor que consideramos esencial para la prevención del maltrato en los niños, resulta prioritario que cuenten con figuras sustitutas que puedan brindarles experiencias de relación constructivas, de modo que ello les sirva como protección para las situaciones posteriores y los ayude a reestructurar y resignificar las vivencias de sus primeros años.

Con respecto a la variable de tipo de maltrato, se observaron diferencias significativas, mediante el S.C., entre el grupo de niños maltratados físicamente y el grupo de niñas abusadas.

Sabemos que el cuerpo brinda un testimonio del maltrato y abuso del que la menor ha sido objeto, conectándola con toda aquella rabia e impotencia y con otros sentimientos desencadenados por este tipo de vivencias. En este sentido, las huellas físicas provenientes del maltrato y abuso generan heridas en la autoimagen y un aumento de la preocupación corporal en las mujeres con respecto a los varones; ya que la sociedad plantea una mayor exigencia y valor a la belleza y la apariencia atractiva en la mujer, lo que influye en la autopercepción (Bordo, 1989).

A partir de las diferencias halladas con relación al tipo de maltrato, coincidimos con Ornduff (1997) al plantear que diferentes tipos de abuso y maltrato influyen y afectan, de manera diferencial, una determinada dimensión del funcionamiento de la personalidad. 
Nos preguntamos qué puede haber generado representaciones más desorganizadas, así como vínculos menos cooperativos y positivos en las niñas. Se propone que tanto la madre como el hijo varón prevén la separación entre ambos, ya que una simbiosis prolongada es amenazante para la masculinidad de este último. De ahí que el niño construya una barrera psíquica que lo proteja del impulso de fusionarse con aquella, para así poder desarrollar su identidad genérica (Stoller y Herdt, 1982). Por otro lado, sostenemos que la mujer desde niña, a diferencia del varón, se define a sí misma en el intercambio y la conexión emocional con el otro (Gilligan, 1982), desarrollando su capacidad de empatía. Al mantener la niña una relación fusionada con la madre, no se encontraría preparada para la separación temprana, afectándole esta de manera más intensa que en el varón.

Adicionalmente, dentro del grupo de niñas, encontramos cierta proporción de casos de abuso sexual cuyo efecto, si bien es intenso como el del maltrato, genera un matiz particular en las que participaron del presente estudio.

Por último, consideramos que existen diferencias de género entre ambos grupos, ya que los varones tienden a mostrarse más impulsivos e inquietos, reaccionando más disruptivamente; mientras que las niñas, reaccionan de manera menos desorganizada que los varones, mostrando una mayor tristeza y ansiedad (Monahon, 1993).

Por lo tanto, planteamos, tanto en función del Rorschach como del MOA, que si bien existen diferencias de género en nuestro estudio, las niñas se encuentran más afectadas, principalmente como consecuencia de haber sido separadas tempranamente de su figura materna y también, debido a que la muestra de niñas cuenta con algunos casos de abuso sexual.

En esa medida, se plantea la necesidad de realizar estudios sistemáticos que establezcan las diferencias que genera el maltrato en ambos géneros en nuestro medio. 
A modo de síntesis, y a través del Rorschach y del MOA, hemos podido comprobar que las variables tipo de maltrato y momento de separación han marcado mayores diferencias en relación con el grupo en general. Así, en lo que se refiere al momento de separación, nuestros hallazgos comprueban que el apego primario brinda el modelo representacional dictando la forma y dirección de las relaciones posteriores. Por otro lado, debemos considerar que los factores externos como el abuso, el rechazo y el maltrato, llevan al desarrollo de un esquema representacional patológico, en el que los objetos son infundidos con atributos negativos en relación con los otros y consigo mismos.

Este trabajo ha intentado brindar un aporte para la psicología clínica al poder estudiar más profundamente las relaciones objetales de estos niños y a partir de este proceso, conocer el vínculo que estos niños establecen con las demás personas. Esto nos ha permitido ver las posibilidades futuras de estos niños y nos ayudaría en la orientación del plan terapeútico.

Esta investigación nos ha permitido demostrar la utilidad y precisión del Psicodiagnóstico de Rorschach, como un método válido para describir las relaciones objetales en niños maltratados e institucionalizados, ya que nos permite conocer y acercarnos al tipo de vínculo que establecen con la figura materna, a partir de la cual el menor logra relacionarse con el entorno. Nos ha parecido particularmente relevante la precisión de este método para discriminar los efectos de la separación temprana de la figura materna, ya que las relaciones tempranas se constituyen como factores causales en el desarrollo de la personalidad posterior (Masling y Bornstein, 1994).

Asimismo, el emplear la Escala de Mutualidad de Autonomía de Urist (MOA), nos ha permitido observar la utilidad de este instrumento en la medición de las relaciones objetales, puesto que nos brinda un aporte sumamente valioso al permitirnos observar que los 
menores aún a pesar de las dificultades experimentadas, logran establecer relaciones adaptativas y sanas con el otro (en un $89 \%$ ). Este dato nos brinda una referencia acerca de los recursos existentes en los niños, que no pueden pasar desapercibidos en el planteamiento de alternativas de atención para con ellos.

La integración de los resultados tanto del MOA como del Rorschach, nos ha permitido observar que se pueden llegar a realizar estudios cuantitativos y cualitativos de las relaciones objetales. Así, el empleo de ambos instrumentos nos ha servido para relacionar los hallazgos, para de esta manera plantear acciones futuras, considerando que las primeras etapas de la vida constituyen la base y cimiento para el desarrollo posterior y que los menores cuentan aún con muchas posibilidades, requiriendo acciones preventivas y terapéuticas de parte nuestra y de la población en general, así como programas organizados que apunten a potencializar los recursos de la infancia y a superar el daño encontrado.

Adicionalmente, nuestro estudio logra brindarnos algunos indicios de la validez convergente del MOA al establecer la relación entre los indicadores de esta escala con los del Rorschach.

Por último, planteamos la enorme trascendencia y el valioso alcance que se tiene, al observar que los menores cuentan con representaciones internas de las relaciones objetales más adaptativas y benignas, que los posibilita a vincularse de manera más diferenciada y con mayor tranquilidad y confianza. Sugerimos la necesidad de conducir estudios en los que, en un primer momento, se evalúe la calidad y el grado de autonomía de las relaciones objetales; para posteriormente, realizar un programa terapéutico que funcione como una experiencia emocional correctiva, a cuyo término sea reevaluada la calidad de las relaciones objetales; y así observar el efecto de la intervención, en un esfuerzo por incrementar sus posibilidades futuras. 
Esperamos que la presente investigación contribuya a la comprensión de los patrones relacionales que guían las relaciones que establecemos tanto con el entorno, como con los otros y con nosotros mismos, para con ello lograr una comprensión más completa e integral de la dinámica del maltrato, integrando las sensaciones dejadas en el menor a partir de esta vivencia, así como los efectos que este puede generar en el funcionamiento yoico y en las defensas que el niño emplea para mantener cierto equilibrio interno.

Apuntamos por ello, a lograr una mayor conciencia de la necesidad de programas preventivos y de promoción de salud así como terapeúticos desde la Psicología Clínica en especial en la Psicología del Desarrollo, que nos brinda el marco referencial dentro del cual podemos reconocer aquellos recursos y fortalezas presentes en nuestra infancia y que les brinda una esperanza para crecer y desarrollarse, actualizando estos recursos y además buscando lograr y brindar un mayor aporte a estos menores para elaborar la situación traumática sufrida, permitiéndoles una comprensión de la situación que los ayude a no repetir estos patrones de relación que tienden a reeditarse, y así contribuir a llevar a su finalización al temido círculo vicioso del maltrato.

\section{Referencias}

Bordo, S. R. (1989). The body and the Reproduction of Femininity: A Feminist Appropriation of Foucalt. En Gender/body/knowledge: Feminist reconstruction of being and knowing. Nuevo Brunswick: Ruitgers University Press.

Exner, J. (1994). El Rorschach: Un Sistema Comprehensivo. Fundamentos Básicos (vol. 1). Madrid: Psimática.

Garrido-Lecca, M. (1999). Los niños ante la violencia: Sus relaciones objetales. Ponencia presentada en el XVI Congreso Internacional de Rorschach y Técnicas proyectivas, Amsterdam. 
Gilligan, C., Lyons, N. y Hanmer, T. (1982). In a different voice: Psychological theory and women's development. Cambridge. MA: Harvard University Press.

Greenberg, J., y Mitchell, S. (1983). Object relations in psychoanalytic theory. Cambridge, MA: Harvard University Press.

Kelly, F. D. (1999). The Psychological assessment of abused and traumatized children. Mahwah, NJ: Erlbaum.

Kernberg, O. (1976). Object-relations theory and clinical psychoanalysis. Nueva York: Aronson.

Levine, L. y Tuber, S. (1993). Measures of mental representation: clinical and theoretical considerations. Bulletin of the Menninger Clinic, 57, 69-87.

Lynch, M., y Cicchetti, D. (1991). Patterns of relatedness in maltreated and nonmaltreated children: Connections among multiple representational models. Developmental Psychopathology, 3, 207-226.

Masling y Borstein, R. F. (1994). Empirical perspectives on object relations theory. Washington, DC: American Psychological Association.

Meyer, J. y Tuber, S. (1989). Intrapsychic and behavioral correlates of the phenomena of imaguinary companions in young children. Psychoanalytic Psychology, 6, 151-168.

Monahon, C. (1993). Children and Trauma. Nueva York: Lexington Books.

Ornduff, S. R. (1997). TAT assessment of object relations: implications for child abuse. Bulletin of the Menninger Clinic, 61, 1-15.

Pine, F. (1990). Drive, ego, object and self: A synthesis for clinical work. Nueva York. Basic Books.

Rivera, S. (2000). Relaciones objetales en niños maltratados y abusados a través del Psicodiagnóstico de Rorschach (Exner) y la Escala de Mutualidad de Autonomía (Urist). Tesis de Licenciatura en Psicología no publicada, Pontificia Universidad Católica del Perú, Lima.

Rosegrant, J. (1984). Rorschach object relations and fantasy themes 
Relaciones objetables en niños maltratados y abusados a través del Rorschach y del MOA

incorrectly scored as determinants. Journal of Personality Assessment, 48, 467-475.

Ryan, R. M., Avery, R. y Grolnick, W. S. (1985). A Rorschach assessment of children's mutuality of autonomy. Joumal of Personality Assessment, $49,6-12$.

Smith, B. (1994). Object Relations Theory and the Integration of Empirical and psychoanalytic Approaches to Rorschach Interpretation. Rorschachiana, 19, 61-77.

Spector, P. (1992). Summated rating scale construction: an introduction. Newbury Park: Sage.

Stoller, R. y Herdt, G. (1982). The development of masculinity: A crosscultural contribution. Journal of the American Psychoanalytic Association, 30 (1), 29-59.

Urist, J. (1977). The Rorschach Test and the assessment of object relations. Journal of Personality assessment, 41, 3-9.

Valeros, J. A. (1997). El jugar del analista. Buenos Aires: Fondo de Cultura Económica. 